\title{
EFECTO DE LOS ÁCIDOS GRASOS OMEGA-3 EN INDIVIDUOS CON OBESIDAD: REVISIÓN SISTEMÁTICA.
}

\author{
EFFECT OF OMEGA-3 FATTY ACIDS IN INDIVIDUALS WITH OBESITY: A SYSTEMATIC REVIEW. \\ Longoria-Oyervidez Miguel Eduardo*, Treviño-Casanova Karina Lizbeth*, Sánchez-Peña María Alejandra*, \\ Cuellar-Robles Sofía*, Nava-González Edna Judith*.
}

*Universidad Autónoma de Nuevo León, Facultad de Salud Pública y Nutrición. México.

\begin{abstract}
RESUMEN
Introducción: La obesidad hoy en día es un grave problema de salud pública a nivel mundial, nacional y local, por lo que se ha estudiado el papel de los ácidos grasos omega-3 en la regulación del peso corporal. Objetivo: Conocer el efecto benéfico de la suplementación con omega-3 en seres humanos con obesidad. Material y Método: Se realizó una búsqueda en cuatro bases de datos en línea donde el tamaño de muestra fue de 34 a 154 participantes, todos los participantes comprendían con un IMC $>30$ y $<40 \mathrm{~kg} / \mathrm{m} 2$ y una edad adulta promedio de 18 a 70 años. La duración de la intervención con suplementación de omega-3 fue de 10 a 30 semanas, utilizando una dosis de $300 \mathrm{mg}$ a 4 gramos. Resultados: La suplementación con los ácidos grasos omega-3, durante 10 a 30 semanas con una dosis de 1 a 3 gramos, podrían ayudar a mejorar el índice de masa corporal (IMC) y el índice cintura-cadera (ICC), al igual que la modulación inflamatoria en las adipocinas IL8 y la proteína C reactiva. Conclusiones: La evidencia de la eficacia de la suplementación con los ácidos grasos omega-3 para la pérdida de peso no es sólida, por lo que es importante continuar investigando y revisando sus efectos. Las limitaciones del estudio: número limitado de estudios, además las investigaciones se realizaron en modelos animales.

Palabras Clave: obesidad, ácidos grasos omega 3, suplementos dietéticos.
\end{abstract}

\section{ABSTRACT}

Introduction: Obesity today is a serious public health problem at the global, national and local level, for which the role of omega- 3 fatty acids in the regulation of body weight has been studied. Objective: To know the beneficial effect of omega-3 supplementation in obese humans. Material and method: A search was carried out in four online databases where the sample size was 34 to 154 participants, all participants had a BMl $>30$ and $<40 \mathrm{~kg} / \mathrm{m} 2$ and an average adult age of 18 to 70 years. The duration of the intervention with omega-3 supplementation was 10 to 30 weeks, using a dose of $300 \mathrm{mg}$ to 4 grams. Results: Supplementation with omega-3 fatty acids, for 10 to 30 weeks with a dose of 1 to 3 grams, could help improve body mass index (BMI) and waist-hip index (ICC), as well as than inflammatory modulation in IL8 adipokines and C-reactive protein. Conclusions: The evidence for the efficacy of omega-3 fatty acid supplementation for weight loss is not strong, so it is important to continue researching and reviewing its effects. Study limitations: limited number of studies, in addition to research conducted in animal models.

Key words: obesity, omega 3 fatty acids, dietary supplements.

Correspondencia: Edna Judith Nava-González edna.navag@uanl.mx

Recibido: 21 de septiembre 2021, aceptado: 06 de diciembre 2021

(C)Autor2022

(c) (i)

DOI: https://doi.org/10.29105/respyn21.1-4

Citation: Longoria-Oyervidez M.E., Treviño-Casanova K.L., Sánchez-Peña M.A., Cuellar-Robles S., NavaGonzález E.J. (2022) Efecto de los ácidos grasos Omega-3 en individuos con obesidad: Revisión Sistemática. Revista Salud Pública y Nutrición, 21 (1), 28-35. 


\section{Introducción}

La prevalencia de obesidad a nivel mundial trae consigo un enorme riesgo de enfermedades metabólicas y cardiovasculares, predisponiendo a un estado pro-inflamatorio (Castellanos T \& Rodríguez D, 2015; Ellulu et al., 2017).

Según datos presentados por el Instituto Nacional de Salud Pública (INSP) en la Encuesta de Salud y Nutrición (ENSANUT) en el año 2018, reportó que, a nivel nacional, el porcentaje de adultos de 20 años y más, con sobrepeso y obesidad fue de $75.2 \%$, porcentaje que en el 2012 fue de 71.3 por ciento (INSP, 2018).

La Asociación Estadounidense de Endocrinólogos Clínicos (AACE) y el Colegio Estadounidense de Endocrinología (ACE) han definido la obesidad como "enfermedad crónica basada en la adiposidad por sus siglas en ingles $\mathrm{ABCD}$ ", este término identifica explícitamente una enfermedad crónica, aludiendo a una base fisiopatológica precisa (Mechanick et al., 2017).

La clasificación actual de Obesidad propuesta por la OMS está basada en el Índice de Masa Corporal (IMC) (Moreno M, 2012). De esta manera, se aplica el término "obesidad" a cualquier persona con una relación peso-talla o IMC de $\geq 30 \mathrm{~kg} / \mathrm{m} 2$. Esta definición ha llegado a confundir por las implicaciones relacionadas con la salud, ya que el diagnóstico se basa únicamente en una medición antropométrica, por lo que el biomarcador de peso y talla no determina la adiposidad por si sola (Mechanick et al., 2017).

Etiología y Fisiopatología de la obesidad: expansión del tejido adiposo.

La obesidad está modulada por factores genéticos, epigenéticos, fisiológicos y de estilo de vida, donde hay elevada ingesta calórica, con bajo gasto energético. El tejido adiposo contiene aproximadamente 600 factores bioactivos considerados adipoquinas, las más estudiadas son la leptina y la adiponectina. Durante la obesidad se observa alteración en la secreción de estas sustancias debido a la inflamación, lo cual conlleva a anomalías metabólicas. En la obesidad como enfermedad crónica, se puede observar mayor infiltración de monocitos (atraídos por sustancias químicas) que a su vez madurarán a macrófagos lo cuales secretarán una gran cantidad de sustancias inflamatorias (Suárez Carmona et al., 2017; Izaola, 2015).

Aspectos de los ácidos grasos omega-3 en la intervención nutricional

Los ácidos grasos omega-3 son ácidos grasos polinsaturados que se encuentran en tres principales formas en los alimentos: ácido eicosapentaenoico (20:5 omega-3, EPA), ácido docosahexaenoico (22:6 omega-3, DHA) y alfa linolénico (18:3 omega-3, aALA) (Castellanos T \& Rodríguez D, 2015).

Hoy en día se conoce del requerimiento de mayores concentraciones de ácidos grasos omega-3, recomendadas para la prevención de enfermedades cardiovasculares y neurodegenerativas, con efecto, antiinflamatorio, aunque aún falta evidencia, se ha motivado al desarrollo de procesos que permiten obtener altas concentraciones de ácidos grasos omega-3 ya sea de EPA, DHA o de ambos. Este proceso se puede realizar de forma química o mediante enzimas; el cual consiste en separar los ácidos grasos de estructura triglicerídica de los aceites marinos, transformándolos en esteres del etanol (Valenzuela B, 2014).

Mecanismo de los omega-3 en la pérdida de peso. Los posibles mecanismos relacionados con el efecto de los omega-3 sobre el peso corporal o la masa de grasa corporal parecen estar asociados con cambios metabólicos relacionados con la obesidad, incluida la modulación del metabolismo de los lípidos, la regulación de las adipocinas (como la adiponectina y la leptina) y la disminución de la inflamación del tejido adiposo (Delpino et al., 2021).

Los ácidos grasos omega-3 son eficaces para proteger contra la obesidad al activar el tejido adiposo pardo (BAT) que ayuda al gasto energético a través de su función termogénica especializada. El EPA aumenta la termogénesis mediante el desacoplamiento de la proteína 1 (UCP-1), esta proteína estimula el consumo de energía y permite la generación de calor (Saini \& Keum, 2018).

Según Faurot et.al (2016) cada vez es más complejo consumir ácidos grasos omega-3 de origen marino, por lo que el uso de suplementos dietéticos es común, su evaluación es desafiante, especialmente entre las poblaciones de minorías étnicas, como los hispanos / latinos. Aún es controversial mediante la evidencia, 
si el consumo de ácidos grasos omega-3 puede influir en el efecto antiinflamatorio en la obesidad.

Se ha estimado el papel de los ácidos grasos omega3 de cadena larga, docosahexaenoico (DHA) y eicosapentaenoico (EPA) en la regulación del peso corporal y efecto antiinflamatorio, por lo que, según la evidencia científica encontrada, el objetivo del presente estudio es revisar la literatura sobre investigaciones que evaluaron el efecto benéfico de la suplementación con omega-3 en seres humanos con obesidad.

\section{Metodología}

Para resolver el problema o tema de revisión se elaboró la pregunta PICO considerando los siguientes elementos: $\mathrm{P}$; (paciente o problema): estudios con personas mayores o igual a 18 años y un índice de masa corporal mayor o igual a $30 \mathrm{~kg} / \mathrm{m} 2$. I; (intervención): suplementación con los ácidos grasos omega-3. C; (Comparación): con grupo placebo. O; (outcome o resultado): donde se muestren resultados en los parámetros antropométricos (peso, masa grasa, masa libre de grasa, circunferencia de cintura e ICC), perfil bioquímico y antinflamatorio en adultos con obesidad. Por lo tanto, la pregunta PICO fue la siguiente: ¿El omega 3 tiene un efecto benéfico en la obesidad? Para contestar esta pregunta, el presente estudio se ajustó a la declaración PRISMA para revisiones sistemáticas y metanálisis.

Estrategia de búsqueda

Se realizó la búsqueda de ensayos clínicos en buscadores como Pubmed $\AA$, Science Direct $₫$, Google académico $®$ y Cochranelibrary $®$. La información que se utilizó para esta revisión se identificó en artículos acerca de los ácidos grasos omega-3 y su efecto en la obesidad. Las palabras claves que se utilizaron fueron: "obesidad", "omega3", "suplementación”, "PUFAS”, "Perfil de lípidos”, "Inflamación", "Adulto", y las combinaciones entre estas en idioma inglés y español.

\section{Selección de estudios}

En esta revisión basada en la metodología de las revisiones Cochrane, se creó una base de datos con todos los artículos identificados en el software Excel® versión 2013. Se consideraron los estudios realizados en humanos (mayores o igual a 18 años y un índice de masa corporal mayor o igual a 30 $\mathrm{kg} / \mathrm{m} 2$ ), y solo cuando se administró los ácidos grasos omega-3 como suplemento. De ellos se incluyeron todos los que cumplían los siguientes criterios: 1) Los que se reportaron como prospectivos, aleatorios (paralelos o transversales), ciegos (dobles o únicos) y de control, con tamaño de la muestra mayor a 30. 2) suplementación con los ácidos grasos omega-3 (proporción de EPA y DHA) o ser grupo control en un periodo mayor de 3 semanas; 3) los que reportaron al menos uno de los siguientes parámetros de composición corporal incluyendo peso corporal, IMC, circunferencia de cintura e índice de cintura-cadera (ICC); 4) Perfil bioquímico enfocado en perfil de lípidos e inflamatorio. Fueron excluidos todos los que no cumplieron los parámetros de selección y los estudios con enfoque a pacientes con obesidad en la unidad de cuidados intensivos.

El proceso de cribado fue realizado individualmente según los criterios de inclusión y exclusión previamente definidos. En caso de desacuerdo, los autores discutieron para establecer un consenso. Primero, cada autor seleccionó los títulos de los artículos de interés de forma independiente. La segunda etapa consistió en la lectura de los resúmenes seleccionados; posteriormente, los artículos se leyeron en su totalidad. Finalmente, también se revisaron las referencias de todos los artículos leídos en su totalidad.

Evaluación de la calidad

La evaluación de la calidad de los artículos se realizó utilizando la herramienta de evaluación de riesgos Cochrane (Higgins JPT, et al). Esta escala evalúa siete ítems, y cada ítem debe clasificarse como de bajo riesgo, alto riesgo o riesgo de sesgo poco claro. Los siete ítems son: 1- generación de secuencia aleatoria; 2- ocultación de la asignación; 3cegamiento de pacientes y personal; 4- cegamiento de la evaluación de resultados; 5- datos de resultado incompletos; 6- notificación selectiva; 7- otro sesgo. Los meta análisis fueron revisados individualmente y analizados para obtener los datos puntuales de cambio en los parámetros antropométricos, perfil bioquímico y antinflamatorio

El trabajo fue registrado y revisado por el comité de ética, cumpliendo con la declaración de Helsinki.

\section{Resultados}

De acuerdo a la búsqueda realizada se encontraron 37 artículos en total, que contenían las palabras clave 
(mencionadas en metodología) en el título o abstract de los artículos. En el primer filtro se excluyeron aquellos que en el título o resumen especificaran que eran de revisión sistemática, reporte, critical reviews y que fueran realizados en animales, enfocados en otros grupos de edad (población pediátrica <18 años y/o adultos mayores $>70$ años), por el año comprendido $(<2010)$ y por el tamaño de muestra (<30 participantes). Posterior a este filtro se seleccionaron 14 artículos los cuales fueron analizados y de estos se eliminaron 6 por evaluar ingesta a través de alimentos ricos en omega-3, enfoque en población con sobrepeso e ingesta de cápsulas de omega-3 modificadas con otra sustancia bioactiva. De acuerdo con los criterios de selección 8 artículos cumplieron con la metodología de inclusión (ver figura 1 ).

Figura 1. Estudios obtenidos en bases de datos evaluados para la inclusión.

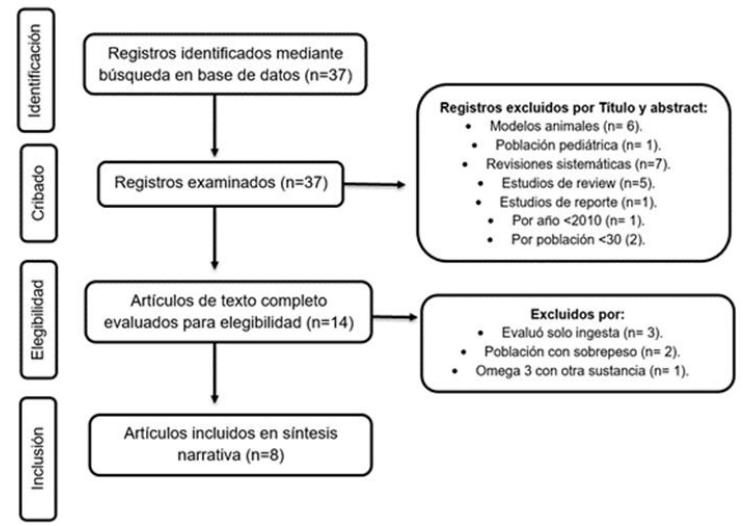

Fuente: Elaboración propia.

En el Consort se incluyen las características de la evaluación y tratamiento de los estudios, los cuales sus años comprendidos fueron del 2010 al 2020, de los artículos que se encontraron $97 \%$ cumplieron con esta característica, encontrándose mayor cantidad de publicaciones del año 2013. El tiempo de publicación abarcó del 2011 al 2018, y los del 2013 con tres artículos. El tamaño de muestra fue de 34 a 154 participantes, todos los participantes comprendían con un IMC $>30 y<40 \mathrm{~kg} / \mathrm{m} 2$ y una edad adulta promedio de 18 a 70 años.

La duración de la intervención con suplementación de omega-3 fue de 10 a 30 semanas. Usando una dosis de omega-3 de $300 \mathrm{mg}$ a 4 gramos como dosis mínima y máxima respectivamente, además con administración de placebos al grupo control en 3 estudios, mientras que en 3 se ofrecieron solo cápsulas de omega-3 y en uno combinado. Todos los artículos evaluaron datos antropométricos, con al menos un parámetro, pruebas de laboratorio como perfil de lípidos, y perfil inflamatorio, otras pruebas más específicas como prueba de tolerancia a la glucosa e índice de calidad del sueño. Para evaluar ingesta se utilizaron frecuencia de alimentos (ver tabla 1).

Efectos de omega-3 en fenotipos antropométricos y de composición corporal

Los artículos que reportaron el efecto de los ácidos grasos omega-3 en los cambios de peso corporal fueron 4, Keshavarz, A y cols., reportaron una pérdida del peso corporal de $3,37 \%(-3,17 \mathrm{~kg})$ y un $4,35 \%(-4,19 \mathrm{~kg})$ para placebo y omega-3, respectivamente. Mientras que en el estudio de DeFina, F. y cols., no se observaron diferencias significativas. Los resultados calculados indicaron que el omega-3 no se asoció con una reducción significativa del peso corporal en comparación con los controles. En el IMC se observaron diferencias significativas en todos los artículos, con una disminución con respecto al valor basal en todos los grupos, en los grupos placebo y a los que se les dio omega-3, siendo más significativo la disminución en la dosis de 1 y 2 gramos.

En total, la investigación en los efectos de omega-3 sobre los cambios en la circunferencia de la cintura no se observó heterogeneidad significativa entre los participantes, mientras que, en el ICC, el tratamiento no se encontró asociado en ninguna forma con las modificaciones en el índice cintura-cadera, dado la reducción proporcional de la cadera.

Keshavarz, A. et al reportó el efecto de omega-3 en la composición corporal encontrándose una reducción de la masa grasa del $8.95 \%(-3,43 \mathrm{~kg})$ y un aumento de la masa libre de grasa del 0,67\% (0,29 $\mathrm{kg}$ ) para el placebo. Para el grupo de omega-3 hubo una reducción tanto en masa grasa de 9,76\% (-3,8 $\mathrm{kg})$ como en masa libre de grasa $0,51 \%(-0,36 \mathrm{~kg})$. Solo González, O. et al. reportó que no se encontró influencia del tratamiento sobre los cambios en la masa libre de grasa observados, los cuales fueron inferiores al $1 \%$ en todos los grupos, y en la masa grasa se observaron diferencias significativas. 


\begin{tabular}{|c|c|c|c|c|}
\hline Autor & $\begin{array}{l}\text { Dosis de } \\
\text { omega } 3\end{array}$ & Duración & Parámetros evaluados & Resultados \\
\hline Munro A. et al. & $\begin{array}{c}1 \text { g de Omega- } \\
3(\mathrm{DHA})+ \\
(\mathrm{EPA})\end{array}$ & 12 semanas & $\begin{array}{l}\text { Diario de alimentos (3 días). } \\
\text { Peso, IMC, CC e ICC, MG, MLG y } \\
\text { MM. Ácidos grasos plasmáticos. } \\
\text { Niveles de TNFa y IL-6. }\end{array}$ & $\begin{array}{l}\text { La suplementación redujo el peso } \\
\text { corporal y de la C.C. Además, hubo } \\
\text { reducción en los triglicéridos, } \\
\text { mientras que no hubo cambios en el } \\
\text { resto de lípidos en sangre. }\end{array}$ \\
\hline \multirow[b]{2}{*}{ DeFina, F. et al } & \multirow{2}{*}{$\begin{array}{c}3.0 \text { g de } \\
\text { Omega- } 3 \\
(E P A)+(D H A)\end{array}$} & \multirow[b]{2}{*}{24 semanas } & $\begin{array}{l}\text { Talla, PA, IMC, CC, y Prueba } \\
\text { psiquiátrica. }\end{array}$ & \multirow{2}{*}{$\begin{array}{l}\text { No hubo diferencia en la pérdida de } \\
\text { peso en ambos grupos con y sin } \\
\text { suplementación. Además, no hubo } \\
\text { cambios en los otros parámetros } \\
\text { evaluados. }\end{array}$} \\
\hline & & & $\begin{array}{l}\text { PCR y hormona tiroidea } \\
\text { estimulante y ácidos grasos. } \\
\text { Prueba de ejercicio en bicicleta. }\end{array}$ & \\
\hline \multirow[b]{2}{*}{$\begin{array}{c}\text { Spencer, M. et } \\
\text { al }\end{array}$} & \multirow{2}{*}{$\begin{array}{c}4 \text { g de Omega- } \\
3 \text { (EPA) }+ \\
\text { (DHA). }\end{array}$} & \multirow[b]{2}{*}{12 semanas } & Cuestionario de alimentarios. & \multirow{2}{*}{$\begin{array}{l}\text { Hubo disminución de triglicéridos en } \\
\text { el grupo suplementado, en el perfil de } \\
\text { lípidos, glucosa, IL-10, IL-12, resistina } \\
\text { y leptina no hubo cambios. }\end{array}$} \\
\hline & & & $\begin{array}{l}\text { Prueba de tolerancia a la } \\
\text { glucosa oral, lípidos séricos, } \\
\text { función tiroidea y laboratorios } \\
\text { de rutina. }\end{array}$ & \\
\hline Kiecolt, K. et al & $\begin{array}{c}0.5 \mathrm{~g} \text { de } \\
\text { Omega- } 3 \text {, de } \\
347,5 \mathrm{mg} \text { de } \\
\text { ácido (EPA) y } \\
58 \mathrm{mg}(\mathrm{DHA})\end{array}$ & 16 semanas & $\begin{array}{l}\text { Talla y peso. Ácidos grasos } \\
\text { plasmáticos, estado de ánimo y } \\
\text { citocinas IL-6 y TNF-a. Diámetro } \\
\text { abdominal, cuestionario de } \\
\text { Frecuencia alimentaria e índice } \\
\text { de calidad del sueño. }\end{array}$ & $\begin{array}{l}\text { No hubo diferencias significativas en } \\
\text { ningún parámetro salvo en la IL-6. }\end{array}$ \\
\hline $\begin{array}{c}\text { Keshavarz, A. et } \\
\text { al }\end{array}$ & $\begin{array}{c}\text { Omega-3 } \\
\text { (cada cápsula } \\
180 \mathrm{mg} \text { de EPA } \\
\text { y } 120 \mathrm{mg} \text { de } \\
\text { DHA) }\end{array}$ & 12 semanas & $\begin{array}{l}\text { Peso, talla, IMC, ICC la MG y MM } \\
\text { por InBody } 270 \text { y el inventario de } \\
\text { depresión de Beck. }\end{array}$ & $\begin{array}{l}\text { Hubo reducción de peso y del estado } \\
\text { de depresión. Sin cambios en el } \\
\text { apetito y en el autocontrol de } \\
\text { alimentos. Además, hubo un retorno } \\
\text { de peso en el grupo suplementado. }\end{array}$ \\
\hline $\begin{array}{l}\text { González, O. et } \\
\text { al. }\end{array}$ & $\begin{array}{c}1 \text { g y } 2 \text { g por } \\
\text { día de Omega- } \\
3\end{array}$ & 12 semanas & $\begin{array}{l}\text { Peso, IMC, índice cintura cadera } \\
\text { y distribución grasa. Se midió: } \\
\text { masa grasa (\%), masa magra (Kg.) } \\
\text { y peso (Kg.) en forma basal, al } \\
\text { mes, a los dos y tres meses. }\end{array}$ & $\begin{array}{l}\text { Hubo reducción de peso en todos los } \\
\text { grupos, por lo que se observó en toda } \\
\text { una disminución del IMC. Además, no } \\
\text { se encontró influencia del } \\
\text { tratamiento en la masa magra, grasa e } \\
\text { ICC. }\end{array}$ \\
\hline Vors, C. et al. & $\begin{array}{c}1 \mathrm{~g} \text { de omega- } \\
3 \text { por día que } \\
\text { proporcionab } \\
\text { an: } 2,7 \mathrm{~g} / \mathrm{d} \\
\text { EPA, } 2,7 \mathrm{~g} / \mathrm{d} \\
\text { DHA }\end{array}$ & 10 semanas & $\begin{array}{l}\text { IMC, circunferencia de cintura. } \\
\text { Concentraciones séricas de } \\
\text { colesterol total, triglicéridos, } \\
\text { HDL y glucosa. Extracción de } \\
\text { ARN de sangre total y PCR. }\end{array}$ & $\begin{array}{l}\text { Hubo aumento de las } \\
\text { concentraciones séricas de } \\
\text { adiponectina y disminución de los } \\
\text { niveles séricos de IL-18. Hubo } \\
\text { cambios en las concentraciones } \\
\text { séricas de PCR. Además, se redujo la } \\
\text { PCR plasmática. }\end{array}$ \\
\hline Allaire, J. el al. & $\begin{array}{l}3 \mathrm{~g} / \mathrm{d} \text { de } \\
\text { Omega-3 }\end{array}$ & $\begin{array}{c}10 \text { semanas } \\
\text { cada fase } \\
\text { (3) }\end{array}$ & $\begin{array}{l}\text { Circunferencia de cintura y } \\
\text { cadera. Composición corporal } \\
\text { con absorciometría de rayos X } \\
\text { de energía dual. Cuestionario de } \\
\text { frecuencia de alimentos. } \\
\text { Colesterol total, TG y las HDL. }\end{array}$ & $\begin{array}{l}\text { Hubo cambio en las concentraciones } \\
\text { de C reactiva, además del índice de } \\
\text { omega- } 3 \text { fue mayor en hombres. }\end{array}$ \\
\hline
\end{tabular}


Efectos de la omega-3 en el perfil de lípidos y citocinas proinflamatorias

En total, la investigación en los efectos de omega-3 en el grupo de suplementación y placebo sobre los cambios en el perfil de lípidos, reportaron una reducción significativa en los triglicéridos, mientras que en el resto no hubo no se observaron diferencias de gran impacto, además los fosfolípidos plasmáticos aumentaron significativamente gracias al DHA, encontrándose el efecto contrario del EPA.

No se observaron cambios en los niveles de IL-10, IL-12, resistina, PAI-1 o leptina. Mientras que hubo efectos de significativos sobre las citocinas TNF-a, IL- 6 y IL-18. En la PCR hubo un cambio importante con la suplementación de omega-3, siendo uno de sus principales efectos en el organismo.

Otros parámetros estudiados de la suplementación con omega-3

No se observaron cambios en la glucosa en ayunas o en 2 h, la sensibilidad a la insulina (SI) o la secreción de insulina. El sueño y el ejercicio no mostraron cambios diferenciales en grupos placebo o de omega3 (Spencer, M. et al; Keshavarz, A. et al).

\section{Discusión \\ Aunque los estudios con animales han demostrado que la ingesta dietética de los ácidos grasos omega-3 puede reducir el peso corporal en roedores con obesidad y puede reducir la acumulación de grasa corporal, particularmente la grasa visceral con un consumo de dieta alta en grasas, la eficacia de la suplementación con omega-3 como complemento de la pérdida de peso en humanos no es sólida.}

En este artículo de revisión sistemática, se investigó el efecto benéfico de la suplementación con omega3 en seres humanos con obesidad, en 8 artículos originales donde se usó placebo y suplementación en adultos con obesidad.

En el artículo de Spencer, M. y cols., mencionan que el tratamiento con omega-3 tiene efectos beneficiosos directos sobre el fenotipo adiposo y que un tratamiento más prolongado puede conducir a una mejor función metabólica. En esta revisión sistemática, se encontró resultados significativos en los cambios de masa grasa, en donde se observó una disminución de ésta a comparación de los grupos control con administración de placebos. Asimismo, se observó una disminución en la masa libre de grasa en algunos artículos. Sin embargo, no se observaron cambios significativos en la masa magra.

En cuestión del perfil de lípidos, se reportó reducción significativa en triglicéridos, no obstante, no se observó ningún cambio significativo. Hubo un aumento de los fosfolípidos plasmáticos debido al consumo de DHA, sin embargo, tuvo el efecto contrario al consumir EPA.

En artículos previamente mencionados señalaban que la suplementación con altas dosis de EPA y DHA tiene efectos similares sobre la expresión de varios genes implicados en la regulación de procesos antiinflamatorios y pro inflamatorios en células sanguíneas completas. Los datos también indicaban que los efectos de EPA y DHA sobre la expresión de genes antiinflamatorios pueden ser más consistentes que sus efectos sobre la expresión de genes proinflamatorios.

En esta revisión sistemática no se observaron cambios significativos en las proteínas proinflamatorias, con excepción de la PCR donde hubo una diferencia significativa con la suplementación de omega-3, teniendo un impacto en el estado de inflamación. Aunque los ácidos grasos omega-3 no pueden reemplazar las buenas conductas de salud como el ejercicio, las personas que están en riesgo debido a enfermedades o afecciones inflamatorias establecidas pueden beneficiarse de su uso. Estos datos proporcionan una ventana a las formas en que los ácidos grasos omega-3 pueden afectar el inicio, la progresión y la resolución de la enfermedad.

Du, S. y cols., explicaron que, en total, 21 comparaciones con 1329 sujetos investigaron el efecto del aceite de pescado en los cambios de peso corporal. No se encontró heterogeneidad significativa, lo que quiere decir que no se asoció con una reducción del peso corporal. Además, los resultados calculados indicaron que el aceite de pescado no se asoció con una reducción significativa en el IMC, y además sugirieron que el aceite de pescado podría reducir significativamente la ICC y esto se asoció más cuando se combinó con un programa de pérdida de peso.

Por lo que, a partir de los resultados de este estudio, la mayor limitación fue el reducido número de 
artículos que se logró analizar debido a que los demás estudios que se encontraron fueron excluidos debido a que no cumplían con los requerimientos de inclusión para realizar esta revisión sistemática. Además, la mayoría de las investigaciones se realizaron en modelos animales. Debido a esto se considera que se necesitan más investigaciones a gran escala durante mucho tiempo para determinar conclusiones definitivas o estandarizar la metodología con diseños adecuados.

\section{Conclusiones y recomendaciones}

La evidencia de la eficacia de la suplementación con los ácidos grasos omega-3 como complemento de la pérdida de peso no es sólida, por lo que es importante continuar investigando y revisando sus efectos en conjunto con un régimen de ejercicio y dietético estructurado; puede sugerirse en el tratamiento de la obesidad para mejorar el estado general de salud y modular los procesos inflamatorios y complicaciones asociadas a futuro, siempre y cuando el paciente requiera su uso.

Por lo que es importante continuar con investigaciones en adultos con obesidad que nos ayuden a comprobar el efecto benéfico de este suplemento, además de que la información en humanos es escasa, encontrándose más investigaciones en modelos animales.

\section{Agradecimientos:}

Agradecemos al programa educativo de la Especialidad en Nutriología Clínica de la Facultad de Salud Pública y Nutrición, UANL que pertenece al Programa Nacional de Posgrado de Calidad (PNPC) del Consejo Nacional de Ciencia y Tecnología (CONACYT) por su apoyo en la realización de esta revisión científica en memoria del Dr. José Juan Longoria Garza.

Financiamiento: El presente estudio no tuvo financiación.

\section{Bibliografía}

Allaire, J., et al. (2017). Supplementation with highdose docosahexaenoic acid increases the Omega3 Index more than high-dose eicosapentaenoic acid. Prostaglandins, Leukotrienes and Essential Fatty Acids, 120, 8-14.

https://doi.org/10.1016/j.plefa.2017.03.008.
Alex Buoite Stella, et al. (2018). Update on the Impact of Omega 3 Fatty Acids on Inflammation, Insulin Resistance and Sarcopenia: A Review. International Journal of Molecular Sciences, 19(1), 218.

https://doi.org/10.3390/ijms19010218.

Castellanos T, L., \& Rodriguez D, M. (2015). El efecto de omega 3 en la salud humana y consideraciones en la ingesta. Revista chilena de nutrición, 42(1), 90-95.

https://doi.org/10.4067/S071775182015000100012

DeFina, L. F., et al. (2011). Effects of omega-3 supplementation in combination with diet and exercise on weight loss and body composition. The American Journal of Clinical Nutrition, 93(2), 455-462.

https://doi.org/10.3945/ajcn.110.002741.

Delpino, F. M., Figueiredo, L. M., \& da Silva, B. G. C. (2021). Effects of omega-3 supplementation on body weight and body fat mass: A systematic review. Clinical Nutrition ESPEN, 44, 122-129. https://doi.org/10.1016/j.clnesp.2021.04.023.

Du, S., Jin, J., Fang, W., \& Su, Q. (2015). Does Fish Oil Have an Anti-Obesity Effect in Overweight/Obese Adults? A Meta-Analysis of Randomized Controlled Trials. PLOS ONE, 10(11), e0142652.

https://doi.org/10.1371/journal.pone.0142652.

Ellulu, M. S., Patimah, I., Khaza'ai, H., Rahmat, A., \& Abed, Y. (2017). Obesity and inflammation: the linking mechanism and the complications. Archives of Medical Science, 4, 851-863. https://doi.org/10.5114/aoms.2016.58928.

Faurot, K. R., et al. (2016). Comparison of a Medication Inventory and a Dietary Supplement Interview in Assessing Dietary Supplement Use in the Hispanic Community Health Study/Study of Latinos. Integrative Medicine Insights, 11, IMI.S25587. https://doi.org/10.4137/IMI.S25587.

González-Acevedo, O., et al (2013). Efecto de la suplementación de omega 3 sobre IMC, ICC y composición corporal en mujeres obesas. 
Archivos latinoamericanos de nutrición 63(3): 224-231.

https://www.alanrevista.org/ediciones/2013/3/art $-5 /$

Higgins JPT, et al. The Cochrane Collaboration's tool for assessing risk of bias in randomised trials. BMJ 2011;343 (7829). https://doi.org/10.1136/bmj.d5928.

INEGI-INSP-SSA. (2020). Encuesta Nacional de Salud y Nutrición 2018. https://www.inegi.org.mx/programas/ensanut/20 $18 /$.

Izaola, O. (2015). Inflamación y obesidad (Lipoinflamación). Nutrición Hospitalaria, 6, 2352-2358.

https://doi.org/10.3305/nh.2015.31.6.8829.

Keshavarz, S. A., et al. (2018). Omega-3 supplementation effects on body weight and depression among dieter women with comorbidity of depression and obesity compared with the placebo: A randomized clinical trial. Clinical Nutrition ESPEN, 25, 37-43. https://doi.org/10.1016/j.clnesp.2018.03.001.

Kiecolt-Glaser, J. K., et al. (2012). Omega-3 supplementation lowers inflammation in healthy middle-aged and older adults: A randomized controlled trial. Brain, Behavior, and Immunity, 26(6), 988-995.

https://doi.org/10.1016/j.bbi.2012.05.011.

Manuel Moreno, G. (2012). Definición y clasificación de la obesidad. Revista Médica Clínica Las Condes, 23(2), 124-128. https://doi.org/10.1016/S0716-8640(12)70288-2.

Mark H. Beers M.D, et al. (2011). (2011) Obesity \& the Metabolic Syndrome. The Merck Manual of Diagnosis and Therapy- 19th Ed (pp 108-119).

Mechanick, J. I., et al. (2017). Adiposity-Based Chronic Disease as a new Diagnostic Term: The American Association of Clinical Endocrinologists and American College of Endocrinology Position Statement. Endocrine Practice, 23(3), 372-378.

https://doi.org/10.4158/EP161688.PS.
Munro, I. A., \& Garg, M. L. (2013). Dietary supplementation with long chain omega-3 polyunsaturated fatty acids and weight loss in obese adults. Obesity Research \& Clinical Practice, 7(3), e173-e181.

https://doi.org/10.1016/j.orcp.2011.11.001.

Saini, R. K., \& Keum, Y. (2018). Omega-3 and omega-6 polyunsaturated fatty acids: Dietary sources, metabolism, and significance - A review. Life Sciences, 203, 255-267. https://doi.org/10.1016/j.lfs.2018.04.049.

Suárez Carmona, W., Sánchez Oliver, A., \& González Jurado, J. (2017). Obesidad en México: recomendaciones para una política de Estado. Fisiopatología de la obesidad: Perspectiva actual. Revista chilena de nutrición, 44(3), 226-233. https://doi.org/10.4067/S071775182017000300226.

Spencer, M., et al. (2013). Omega-3 Fatty Acids Reduce Adipose Tissue Macrophages in Human Subjects With Insulin Resistance. Diabetes, 62(5), 1709-1717.

https://doi.org/10.2337/db12-1042.

Valenzuela B, A., \& Valenzuela B, R. (2014). Acidos grasos omega-3 en la nutrición ¿cómo aportarlos? Revista chilena de nutrición, 41(2), 205-211. https://doi.org/10.4067/S071775182014000200012 .

Vors, C., et al. (2017). Inflammatory gene expression in whole blood cells after EPA vs. DHA supplementation: Results from the ComparED study. Atherosclerosis, 257, 116-122.

https://doi.org/10.1016/j.atherosclerosis.2017.01 .025 . 